Annuaire suisse de politique de développement

27-2 | 2008

Migration et développement: un mariage arrangé

\title{
Migration et développement: les enjeux d'une relation controversée
}

Denise Efionayi-Mäder, Gérard Perroulaz et Catherine

Schümperli Younossian

\section{(2) OpenEdition}

Édition électronique

URL : http://journals.openedition.org/aspd/176

DOI : 10.4000/aspd. 176

ISSN : 1663-9669

Éditeur

Institut de hautes études internationales et du développement

Édition imprimée

Date de publication : 1 décembre 2008

Pagination : 11-20

ISBN : 978-2-940415-07-6

ISSN : 1660-5934

Référence électronique

Denise Efionayi-Mäder, Gérard Perroulaz et Catherine Schümperli Younossian, « Migration et développement: les enjeux d'une relation controversée ", Annuaire suisse de politique de développement [En ligne], 27-2 | 2008, mis en ligne le 18 mars 2010, consulté le 10 décembre 2020. URL : http:// journals.openedition.org/aspd/176 ; DOI : https://doi.org/10.4000/aspd.176 


\title{
Migration et développement: les enjeux d'une relation controversée
}

\author{
Denise Efionayi-Mäder, Gérard Perroulaz \\ et Catherine Schümperli Younossian*
}

D

epuis une trentaine d'années, les migrations internationales se sont intensifiées et complexifiées à l'échelle planétaire. Elles sont concomitantes à la mobilité croissante des marchandises, des services, des capitaux et de l'information, au niveau non seulement régional, mais aussi intercontinental. Face à l'augmentation des inégalités mondiales, le phénomène migratoire devient désormais indissociable des questions liées au développement des pays du Sud. Aux écarts de revenus, présents aussi bien à l'intérieur qu'entre les pays, s'ajoutent les différences en termes sociaux, juridiques et sanitaires.

Au niveau mondial, on estime à près de 200 millions le nombre de personnes ne vivant pas dans le pays de leur naissance pour plus d'une année ${ }^{1}$; près de la moitié d'entre elles sont des femmes. En Suisse, un habitant sur trois a des origines étrangères par ses parents et un quart de la population active ne possède pas de passeport suisse. Non seulement ces personnes occupent les secteurs du travail négligés par les autochtones, mais elles apportent également des qualifications professionnelles recherchées au niveau national.

L'intégration des étrangers et l'immigration irrégulière occupent aujourd'hui une place importante dans les débats publics et cristallisent les oppositions politiques. Toutefois, la migration ne peut être réduite à ces aspects; elle est aussi synonyme de transferts de savoir, d'expériences, d'apports culturels et de développement dans l'ensemble des pays du Sud et du Nord. Les liens entre développement et migration ont, depuis longtemps, fait l'objet de la recherche (en migration), mais ce thème a récemment connu un regain d'intérêt et s'est imposé dans l'agenda

* Denise Efionayi-Mäder, responsable de projet et directrice adjointe du Forum suisse pour l'étude des migrations et de la population (SFM), Université de Neuchâtel.

Gérard Perroulaz, chargé de recherche à l'Institut de hautes études internationales et du développement (IHEID), Genève.

Catherine Schümperli Younossian, directrice du comité de rédaction de l'Annuaire suisse de politique de développement et chargée d'enseignement à l'IHEID.

Le dossier de l'édition 2008 a fait l'objet d'une fructueuse collaboration entre l'Institut de hautes études internationales et du développement (IHEID) à Genève et le Forum suisse pour l'étude des migrations et de la population (SFM) de l'Université de Neuchâtel. Grâce aux réseaux de chacune des deux institutions, il a été possible d'identifier des auteurs de référence issus du monde universitaire, d'organisations internationales, de l'administration fédérale ainsi que d'organisations non gouvernementales. Le comité de rédaction de l'Annuaire tient à remercier très chaleureusement Denise Efionayi-Mäder et Alessandro Monsutti, chargé de cours à l'IHEID, pour leur appui sans faille de la conception à la réalisation de cet ouvrage, garantissant la validité scientifique des contributions retenues. Nous remercions également les évaluateurs des articles, parmi lesquels Reynald Blion, Gianni D’Amato et Etienne Piguet.

1 Selon la définition des Nations unies, un migrant est une personne qui quitte son pays pour une durée d'un an au minimum, avec l'intention de s'établir à l'étranger à long terme. 
politique des Nations unies et des organisations internationales. Les controverses nationales autour des politiques migratoires et de coopération ont favorisé cette émergence.

Le présent dossier de l'Annuaire suisse de politique de développement se propose d'éclairer les thèmes évoqués sous l'angle de la recherche académique et de l'analyse des politiques publiques, sur le plan tant national qu'international. Etudes de cas, témoignages et opinions viennent illustrer les articles théoriques afin de stimuler le débat et la réflexion.

Dans l'article introductif du dossier, Alessandro Monsutti présente les différentes approches théoriques en sciences sociales qui, dès l'après-guerre, structurent la réflexion sur la relation entre migration et développement. Il montre que les positions ont oscillé entre l'optimisme lié à la théorie de la modernisation dans les années 1950 et 1960 et le scepticisme inspiré par la théorie de la dépendance dans les années 1970 et même 1980. Il s'agit ainsi de mettre en perspective l'appréciation positive des effets des migrations sur le développement du Sud, appréciation qui semble prévaloir depuis quelques années sur la scène politique. L'intérêt contemporain pour les transferts de fonds des migrants permet de considérer la mobilité de façon constructive, mais le danger d'une instrumentalisation politique est toutefois possible. Selon l'auteur, célébrer sans ambages les migrations internationales et les transferts de fonds comme la nouvelle solution du sous-développement des pays du Sud dénote d'une absence de perspective historique et théorique. Alors que la Commission mondiale sur les migrations internationales a souligné la nécessité d'une coordination interétatique accrue, les Etats s'attachent en effet au bastion de souveraineté que représente le contrôle des flux migratoires sur leurs territoires et peinent à s'entendre pour mettre sur pied un régime global visant à gérer la question (les accords de coopération qui existent portent essentiellement sur des questions sécuritaires).

Pourtant, la connaissance du lien entre migration et développement a considérablement progressé. Grâce aux études qui se sont multipliées ces dernières années, on commence à connaître les conséquences des mouvements migratoires sur les pays d'origine, de transit et d'accueil et on cerne de mieux en mieux, et dans toute leur complexité, les effets du développement sur les flux migratoires. Si la coopération peut, dans un premier temps, prévenir l'émigration liée à des situations de pauvreté majeure - par exemple dans le cas de l'exode rural -, le développement économique renforce parfois la pression migratoire à moyen terme avant de contribuer à sa diminution à plus long terme (migration hump). Ainsi, si les visions de la coopération au développement et de la politique migratoire peuvent paraître antagonistes dans une perspective à moyen terme, ces optiques divergentes sont susceptibles de s'accorder si l'on se place dans une perspective de longue durée.

Transferts de fonds et développement: espoirs déçus?

La relation entre migration et développement s'est imposée par l'intérêt croissant qui entoure les transferts ou envois de fonds (remittances en anglais) des migrants, ainsi que leurs impacts sur les économies locales des pays d'origine. L'étude des flux financiers montre que les sommes envoyées par les migrants 
dépassent de loin les montants investis par l'aide publique au développement, et cela sans tenir compte des flux informels et de marchandises, qui ne sont pas négligeables. Pour 2006, la Banque mondiale évalue les remittances à 206 milliards de dollars, alors que l'aide publique au développement des pays de l'Organisation de coopération et de développement économiques (OCDE) se monte à 104 milliards de dollars. Dans sa contribution, Philippe Wanner rappelle toutefois les difficultés méthodologiques liées à la définition des remittances, ainsi que l'insuffisance des données statistiques, appelant donc à la prudence dans l'interprétation des chiffres. Au-delà de la question quantitative, l'auteur s'interroge sur deux dimensions: l'impact des envois de fonds sur le développement des pays du Sud et ses différents modes de transfert. Ces questionnements trouvent des éléments de réponse par le biais des études de cas présentées par Claude Auroi, Alessandro Monsutti et Matthias Lerch.

Dans quelle mesure les transferts de fonds permettent-ils de lutter contre la pauvreté et favorisent-ils des investissements productifs dans les pays du Sud? $P$. Wanner nous rappelle que la littérature se limite à décrire et interpréter les effets observés empiriquement dans des pays concernés par l'émigration, sans tenir compte des coûts induits. S'il reconnaît aux envois de fonds un potentiel, il constate que ceux-ci ne contribuent pas toujours d'une manière optimale au développement des pays pauvres. Ils peuvent même provoquer, à l'intérieur des pays de destination des fonds, de profondes inégalités entre ceux qui en bénéficient et ceux qui n'ont pas ce privilège. L'auteur rappelle ainsi qu'il est souvent reproché aux bénéficiaires de ne pas investir dans des activités génératrices d'emplois ou de richesse.

Claude Auroi illustre la relation entre les transferts de fonds des migrants et le développement économique local et régional en Amérique latine. Après avoir apprécié le volume des envois de fonds en direction de l'Amérique latine et précisé leur importance relative pour l'économie de quelques pays de la région, l'auteur s'intéresse à leur utilisation. Son diagnostic rejoint celui de $P$. Wanner: les dépenses des familles de migrants sont principalement des dépenses de consommation. Mais il relativise ce constat négatif en soulignant que les dépenses choisies par les familles de migrants sont «cohérentes par rapport à leur avenir» et «constituent aussi une prévoyance et sécurité sociale que l'Etat ne peut fournir en Amérique latine».

$P$. Wanner et $C$. Auroi relèvent tous deux la difficulté d'inscrire des initiatives «privées» d'envoi d'argent dans des projets collectifs, tout en reconnaissant la nécessité de développer des mesures incitatives pour mieux exploiter le potentiel des transferts de fonds, à l'exemple du programme Tres por uno mis en œuvre au Mexique et au Salvador ${ }^{2}$.

Le choix des modes de transfert révèle des aspects importants de la réalité socio-économique des migrants, d'où l'existence de nombreuses études de cas pour en comprendre les mécanismes. $P$. Wanner rappelle que ceux-ci dépendent principalement des structures bancaires des pays de départ et, surtout, de destination. Dans certains pays du Sud, l'implantation d'institutions bancaires fait cruellement défaut, en particulier dans les régions rurales. Cela favorise

2 Pour chaque dollar investi par les migrants dans des projets d'intérêt public, 3 dollars sont alloués par les gouvernements locaux, régionaux et fédéraux. 
l'émergence d'acteurs financiers spécifiques, à l'instar de Western Union, qui dispose de 170000 agences à travers le monde. Mais les coûts de transaction qu'ils facturent sont relativement élevés au vu des sommes transférées. Selon les estimations de l'auteur, jusqu'à $80 \%$ des transferts restent informels dans certains pays. Pour illustrer cette réalité, l'ouvrage présente deux études de cas, l'une relative aux transferts de fonds vers l'Afghanistan (Alessandro Monsutti) et l'autre à ceux de la Suisse vers la Serbie (Mathias Lerch).

\section{De la fuite à la circulation des cerveaux}

Les débats au sein des organisations internationales se sont largement focalisés sur les envois de fonds, mais les chercheurs ont également porté leur attention sur les transferts de connaissances, principalement sous l'angle du recrutement des travailleurs et de la formation. Si la fuite des cerveaux vers les pays du Nord est une réalité - qui concerne surtout les pays les plus pauvres -, la migration des spécialistes, de même que l'internationalisation de leur formation, peuvent présenter une chance, aussi bien pour les pays d'origine que pour les pays d'accueil. Jean-Baptiste Meyer décrit l'émergence des diasporas ${ }^{3}$ scientifiques transnationales, susceptibles de limiter les flux Sud-Nord des talents. Ces réseaux du savoir, souvent organisés sur une base associative, peuvent prendre de multiples formes, à la fois, virtuelles et concrètes: elles vont de l'échange d'information spécialisée jusqu'à la réalisation de projets de développement conjoints, en passant par le tutorat d'étudiants avancés et la participation ponctuelle des expatriés dans les universités de leur pays d'origine. Les données empiriques disponibles montrent qu'une part substantielle des migrants hautement qualifiés désire mettre son expertise à la disposition des pays d'origine. Si l'Inde et la Chine figurent en tête des réseaux diasporiques du savoir, recensés sur Internet, qui œuvrent au développement de leur pays, le phénomène a pris une ampleur non négligeable à l'échelle mondiale.

Diane Duclos met ce processus en exergue dans une étude de cas consacrée aux artistes, journalistes et intellectuels de la diaspora irakienne. Par le biais de la revue littéraire Mesopotamia et de cafés virtuels, les expatriés se servent de la Toile pour débattre de la reconstruction de l'Etat irakien. En raison de l'occupation et des violences quotidiennes, la participation de leurs compatriotes sur place reste limitée, mais D. Duclos et J.-B. Meyer s'accordent pour souligner que les modes de circulation - virtuel, financier et réel - se relaient et se complètent.

\section{Le rôle des diasporas}

Ces échanges transnationaux ont facilité la prise de conscience du rôle des migrants dans le développement de leur propre pays, non seulement sur le plan financier mais aussi dans leur dimension sociale et politique. La migration est l'expression collective ou individuelle de personnes qui s'y engagent et en endossent personnellement les conséquences. Les organisations internationales

3 Ce terme, aux multiples acceptions, est utilisé dans un sens générique; il comprend les membres d'un groupe ethnique ou national qui maintiennent des liens avec leur pays ou région d'origine. 
et de nombreuses ONG actives dans le domaine du développement ont ainsi été amenées de plus en plus souvent à valoriser les ressources humaines et le rôle potentiel des migrants dans ce domaine.

Prenant appui sur différents exemples issus du contexte africain, Marc-Antoine Pérouse de Montclos constate que les émigrés fournissent des modèles de réussite qui inspirent de nouvelles représentations, alimentent les aspirations au progrès et influencent parfois de manière décisive la redistribution du pouvoir dans le pays d'origine. Simultanément, l'exil peut s'accompagner d'une redéfinition des rôles entre hommes et femmes, faciliter l'accès à l'éducation des filles et leur permettre d'échapper à des traditions oppressantes. Mais à l'instar des envois de fonds, qui en Somalie contribuent à la fois à reconstruire les régions ravagées par la guerre et à entretenir les conflits armés, l'apport des diasporas peut aussi bien favoriser la démocratisation et les négociations de paix que renforcer les divisions entre clans ou factions.

Ces effets contrastés, tout à la fois positifs et négatifs pour le pays d'émigration, se reflètent dans le témoignage d'Ibrahima Thioye, qui a quitté la Mauritanie au début des années 1960 pour la France afin d'aider son père malade. I. Thioye déplore le fait que les migrants - comme la plupart des individus, serait-on tenté d'ajouter - ont «une vision uniquement focalisée sur [leur] famille, [leur] maison, [leur] village» et peinent à penser à long terme.

Cette tendance est, selon M.-A. Pérouse de Montclos, renforcée par la précarité économique et juridique que connaissent nombre de migrants. Celle-ci limite leur influence, comme le constatent également Janine Dahinden et Joëlle Moret sur la base d'une analyse diachronique des associations de migrants serbes et albano-kosovares en Suisse. En retraçant l'évolution de l'engagement transnational de ces collectivités, elles constatent que des associations très actives dans le domaine humanitaire - où sont présentes surtout les femmes - et politique durant la période des conflits armés en Serbie et au Kosovo se sont dissoutes ou se concentrent actuellement sur des problèmes d'intégration en Suisse. Mais les deux auteures indiquent également qu'au-delà du profil socio-économique des personnes migrantes, les autorités et les institutions du pays d'accueil aussi bien que du pays d'origine exercent une influence déterminante sur l'orientation et l'effet des activités des associations de migrants.

\section{Vers de meilleures conditions-cadres}

S'il semble désormais indispensable de considérer les migrants comme des acteurs à part entière, aussi bien J.-B. Meyer et M.-A. Pérouse de Montclos que $J$. Dahinden et J. Moret mettent en garde contre la tentation d'ériger les diasporas en agents quasi naturels du développement qui permettraient à eux seuls de faire face à l'échec présumé de la coopération internationale au développement. De manière convergente, les différentes contributions relèvent qu'une implication féconde des migrants dans le développement ne peut se concevoir sans un soutien des pays d'origine et de destination. Face à la complexification des flux migratoires, les approches unilatérales pour réguler les migrations apparaissent de plus en plus inopérantes. Même si les Etats restent très jaloux de leur souveraineté en la matière, ils prennent peu à peu conscience du fait que seule une 
action concertée au niveau international est à même d'aboutir à des solutions constructives, acceptables à la fois pour les pays d'origine, de transit et de destination et les migrants ou migrantes eux-mêmes.

Les orientations que devraient prendre les politiques publiques dans ce domaine font, entre autres, l'objet de l'article de Denis Drechsler et Jason Gagnon. Leur analyse économique souligne qu'il est souvent difficile de démêler clairement les apports de la migration des aspects défavorables pour le développement économique et social d'un pays, car les effets varient selon les contextes et les cycles migratoires. La perte en capital humain due à l'exode des talents, par exemple, peut se muer en gain si les perspectives d'émigration incitent les jeunes à se former, comme le montre Patricia A. Santo Tomas à l'aide de l'exemple des Philippines. Le cas philippin est d'autant plus remarquable que le gouvernement en question a développé une politique transsectorielle à l'égard des migrants, qui s'étend de la formation précédant le départ jusqu'aux mesures de réintégration après le retour, en passant par le soutien des attachés du travail dans les pays de destination. Les démarches entreprises pour passer des accords bilatéraux afin d'améliorer la protection des émigrés méritent également d'être mentionnées. Pourtant beaucoup reste à faire, car ces «travailleurs philippins d'outre-mer», dont aujourd'hui plus de $60 \%$ sont des femmes, n'échappent pas à la précarisation des conditions de travail (travail temporaire et/ou clandestin).

D. Drechsler et J. Gagnon - tout comme P. Santo Tomas - insistent sur le fait, pas toujours perçu par les milieux politiques, que la migration intercontinentale restera une partie intégrante du développement futur à l'échelle mondiale. A condition que l'échange et la mobilité soient encouragés de part et d'autre, les migrants qualifiés, et aussi ceux qui le sont moins, sont susceptibles de contribuer au développement par des investissements, le commerce, ainsi que le transfert de compétences et de savoir-faire social et politique. Dans ce sens, les auteurs recommandent aux Etats de l'OCDE de mieux coordonner les politiques sectorielles, mais aussi de multiplier les partenariats avec les Etats d'origine et d'associer les diasporas aux politiques de développement.

\section{Migration et coopération au développement: un lien ambigu}

L'idée d'associer les migrants - directement ou par le biais d'organisations - au développement économique de leur village ou de leur région est également au cœur de la notion de «codéveloppement», forgée en France. Jérôme Audran démontre comment la politique migratoire française, ancrée dans des préoccupations sécuritaires, s'est peu à peu ouverte à la problématique du développement. La création, en 2007, du Ministère de l'immigration, de l'intégration, de l'identité nationale et du codéveloppement traduit la volonté de Nicolas Sarkozy de créer un lien solide entre politique de développement et contrôle des migrations. Après avoir brossé les principales étapes historiques de l'émergence du concept de «codéveloppement», l'auteur évalue les côtés positifs et négatifs de l'instrumentalisation de la coopération au développement pour lutter contre l'immigration illégale. Malgré cette ambiguité, il conclut que «le codéveloppement offre la possibilité de penser la migration en termes d'intérêts partagés et de renforcer ainsi la coopération Nord-Sud». 
La dernière partie de ce dossier porte sur les politiques menées par la Suisse dans le domaine des liens entre migration et développement. Gianni D'Amato dresse en premier lieu l'historique des politiques migratoire et d'asile en Suisse puis analyse leur évolution, marquée par une politique d'admission différenciée selon le niveau de qualification des personnes (ouverture pour les personnes très qualifiées et celles venant de l'Union européenne, statut de saisonnier autrefois pour les personnes moins qualifiées et frein à l'immigration de personnes peu qualifiées). Il relève la dynamique toujours plus restrictive de la politique de la Suisse.

Le contexte politique national de durcissement de la Loi sur l'asile et de la Loi sur les étrangers n'a pas empêché la recherche d'un débat plus large sur les migrations. La Suisse a poussé les réflexions sur le plan international pour améliorer le dialogue entre pays d'origine des migrants et pays d'accueil; au niveau national, des efforts importants ont été entrepris dès les années 1980 pour une meilleure coordination interdépartementale entre les offices concernés par la migration.

Pietro Mona relève les étapes menant à cette collaboration interdépartementale et souligne la nécessité d'une approche collective pour une politique plus cohérente (whole-of-government approach). Il rappelle qu'avec le lancement de l'Initiative de Berne en 2001, la Suisse a donné une impulsion importante en vue d'une coopération internationale renforcée dans le domaine migratoire, qui a débouché sur la création de la Commission mondiale sur les migrations internationales. Dans son rapport rendu en octobre 2005, la commission traite en priorité du lien entre migration et développement, et la réflexion se poursuit dans le cadre du Dialogue de haut niveau sur les migrations internationales et le développement, mis sur pied en 2006.

Si la Suisse est très active dans les processus internationaux, elle a préalablement mené de nombreux débats au niveau national, notamment au sein de l'administration, à la suite des défis posés par la migration d'asile en provenance de l'ancienne Yougoslavie. Cette région constituait avant l'éclatement des guerres un large réservoir de main-d'œuvre. L'expérience de l'aide au retour, mise en place dès le milieu des années 1990, illustre l'importance d'une coopération concertée entre les divers secteurs politiques (politique extérieure, développement, asile, étrangers, etc.).

Eric Kaser et Saskia Schenker détaillent le fonctionnement de l'aide au retour, cet instrument important de la politique extérieure suisse dans le domaine migratoire. L'aide individuelle à la personne qui doit retourner dans son pays est complétée par des appuis spécifiques (formation, aide dans le domaine de la santé) et, surtout, par une aide structurelle sur place dans les régions concernées, apportée dans le cadre des programmes par pays et assumée par la Direction du développement et de la coopération. L'aide au retour, développée en concertation par plusieurs offices de la Confédération, notamment l'Office fédéral des migrations et la DDC, a bénéficié en dix ans à plus de 65000 personnes, dont 40000 dans les Balkans (surtout en Bosnie-et-Hezégovine et au Kosovo).

Dans une approche plus globale, l'article 100 de la nouvelle Loi sur les étrangers, entrée en vigueur au début de 2008, prévoit que le Conseil fédéral encourage les 
partenariats bilatéraux avec d'autres Etats dans le domaine des migrations. Therese Liechti et Monica Budowski examinent la portée de ces accords. Selon les auteures, trois aspects sont importants dans la mise sur pied des partenariats migratoires. D'abord, le mode de coopération doit permettre à chacun des partenaires de défendre ses propres intérêts. Ensuite, il faut s'entendre sur la migration voulue et la migration indésirable: les priorités varient selon que l'on se concentre sur le renforcement du contrôle pour empêcher la migration non voulue ou sur des mesures destinées à favoriser le potentiel positif des migrations pour le développement d'un pays. Enfin, la collaboration entre les départements est à la fois une condition et la clé de partenariats réussis. E. Kaser et $S$. Schenker relèvent que les enseignements des programmes d'aide au retour menés dans les Balkans peuvent être utiles pour l'établissement du premier partenariat migratoire de la Suisse.

Gérard Perroulaz examine les liens établis en Suisse entre politique de coopération au développement et politique des migrations. $\mathrm{Si}$, au niveau du discours, la coopération suisse accorde une grande importance au thème des migrations et divers offices de la Confédération se sont engagés en faveur d'un dialogue sur le plan international, ce thème a par contre plus de problèmes à se concrétiser au niveau opérationnel, sauf dans l'aide d'urgence. Même si des synergies ont été possibles dans certains pays prioritaires de la DDC, les actions de la coopération suisse sont restées axées essentiellement sur l'aide d'urgence et la lutte contre les effets négatifs des migrations. L'article lance quelques pistes pour promouvoir un rôle plus actif de la coopération au développement et renforcer les effets positifs sur le développement des pays d'origine des migrants.

Christoph Wehrli avance quelques raisons pouvant expliquer l'hésitation de la coopération suisse à s'engager dans l'élaboration de programmes de coopération en matière de migration. Si le phénomène migratoire est régulièrement évoqué dans les messages du Conseil fédéral sur la coopération au développement, les milieux de la coopération comme le monde politique ont été réticents à utiliser une partie du budget de l'aide pour régler des questions relatives à l'asile. Craignant d'être instrumentalisée pour freiner et prévenir les migrations, réticente à l'idée de s'écarter de l'objectif prioritaire d'aider les plus pauvres, la coopération suisse ne s'est pour l'instant guère engagée dans ce domaine.

Toutefois, des ONG de coopération au développement ont récemment engagé une réflexion pour renforcer les synergies entre développement et migration. Yanik Marguerat et Christelle Genoud évoquent les réflexions en cours au sein de la Fédération genevoise de coopération dans ce domaine. Ils constatent cependant que rares sont encore les projets de solidarité et de coopération au développement à intégrer un dialogue entre ONG du Nord, communautés bénéficiaires du Sud et associations de migrants.

$$
* * *
$$

Le fait que la migration fait partie intégrante des processus de développement socio-économique de nos sociétés et que ces derniers sont presque toujours tributaires de migrations, internes ou internationales, a été depuis longtemps établi 
par la recherche, comme l'a récemment rappelé Ronald Skeldon ${ }^{4}$ Les différentes contributions de cet Annuaire présentent quelques-unes des multiples facettes du lien entre les deux phénomènes. D'autres, non moins importantes, n'ont pu être abordées dans ce cadre. Nous pensons notamment aux dynamiques spécifiques qui caractérisent les migrations forcées dans les régions en crise. Les déplacements de populations et leur prise en charge dans des camps accueillant plusieurs dizaines de milliers de personnes ne constituent pas seulement des défis de taille en termes de développement socio-économique et environnemental, mais soulèvent également des questions de sécurité et de stabilité pour les pays concernés, dont certains figurent parmi les plus pauvres de la planète. Pour faire face à ces situations, qui se prolongent parfois pendant plusieurs décennies, il est nécessaire de mieux connaître les conséquences des interventions humanitaires, des politiques migratoires et des politiques de développement. De nombreux acteurs internationaux attirent simultanément l'attention des décideurs sur les effets du changement climatique et des détériorations de l'environnement, qui peuvent également entraîner des migrations involontaires.

L'augmentation et les nouvelles dynamiques des migrations féminines mériteraient un dossier à elles seules, tant leurs implications pour le développement sont cruciales. Il suffit de penser aux innombrables travailleuses migrantes qui s'occupent des enfants et des personnes âgées dans les grandes villes du Nord et du Sud et entretiennent des familles entières, tandis que leurs enfants sont élevés au pays d'origine par d'autres membres de la famille. Bien que ces femmes disposent parfois de bonnes qualifications professionnelles, elles se retrouvent souvent en situation clandestine en dépit de la compétition entre pays développés pour attirer les meilleurs talents. Mais si les facettes les plus problématiques des nouvelles migrations féminines, à l'instar de la traite des êtres humains, ont attiré l'attention de la recherche, les potentialités liées à la mobilité indépendante des femmes ont encore été peu investiguées.

Pour permettre aux lectrices et lecteurs d'aborder des thèmes qui n'ont pas pu être approfondis, nous avons sélectionné quelques portes d'entrée sur Internet qui renvoient à des sites spécialisés. Les sites proposés par Chantal Hebeisen et Martine Schaer dans le Point d'appui ne constituent pas une liste exhaustive, mais présentent un choix d'institutions politiques, non gouvernementales ou scientifiques, sur les plans suisse et international.

Pour conclure cette introduction, nous pouvons relever que le principal mérite du regain d'intérêt pour les liens entre migration et développement est de rappeler que la migration a accompagné le développement tout au long de l'histoire humaine. Aujourd'hui comme hier, il serait illusoire de vouloir contrôler, voire bloquer la migration internationale. Il convient au contraire de prendre acte de cette réalité et de reconnaître que la migration internationale peut constituer un réel atout pour le développement des régions d'origine et - à ne pas oublier des pays d'accueil. Les articles présentés dans cet Annuaire soulignent cependant la complexité des liens entre les deux phénomènes. Malgré les discours politiquement intéressés, qui brouillent un peu les pistes, une observation élémentaire s'impose à la lecture des différentes contributions: la migration ne

4 «International Migration as a Tool in Development Policy: A Passing Phase ?», Population and Development Review, vol. 34, n 1, March 2008, pp. 1-18. 
constituera jamais une recette miracle pour servir le développement des pays du Sud, tout comme ce dernier ne pourra pas faire tarir les flux migratoires. Ces constatations vont à l'encontre de certains messages qui tentent plus ou moins explicitement de redorer le blason de deux politiques publiques (migration et coopération au développement) qui ont fait face à de nombreuses contestations durant ces dernières années. Ce n'est donc pas un hasard si la thématique doit son retour sur le devant de la scène à la sphère politique et aux organisations internationales.

Pourtant, force est de constater que le champ d'action en question est assez réfractaire à une intervention politique immédiate et volontariste, pour les raisons déjà évoquées et parce qu'il est mû par des intérêts divergents et régi par les interventions de multiples acteurs. Cette constatation peut nous amener à penser que le mariage arrangé entre les deux politiques ne débouchera jamais sur une union fusionnelle. Cela ne veut évidemment pas dire que les nombreux débats et recherches passés ou en cours sur les rapprochements entre migration et développement soient vains. Bien au contraire, ils apportent une compréhension indispensable de l'articulation des enjeux et de l'interaction entre les différents partenaires. Et tant mieux si les personnes migrantes sont désormais reconnues comme des acteurs à part entière, dont les intérêts sont à prendre en considération, au même titre que ceux des pays d'origine et de résidence. Au regard des résultats présentés, une instrumentalisation de l'un au service de l'autre risque de provoquer des effets qui, à moyen ou à long terme, vont à l'encontre des résultats recherchés. En termes d'action, il s'agit plutôt de créer des plates-formes de concertation et de coordination, qui laissent aux intervenants une marge de manœuvre suffisante pour œuvrer en vue de structures et de conditions-cadres favorables aux échanges. On peut penser que le succès et la durabilité de ce mariage savamment arrangé résideront davantage dans une large panoplie de mesures bien concertées avec tous les intéressés, au sens d'une approche de soft policy, que dans un tournant politique spectaculaire. Voilà qui permettrait aux migrations de déployer des effets propices au développement. 